\title{
Simulation of the Hybrid and Steady State Advanced Operating Modes in ITER
}

\author{
C. E. Kessel ${ }^{1}$, G. Giruzzi ${ }^{2}$, A. C. C. $\operatorname{Sips}^{3}$, R. V. Budny¹, J. F. Artaud ${ }^{2}$, V. Basiuk ${ }^{2}$, F. \\ Imbeaux $^{2}$, E. Joffrin ${ }^{2}$, M. Schneider ${ }^{2}$, M. Murakami ${ }^{4}$, T. Luce ${ }^{5}$, Holger St. John ${ }^{5}$, T. Oikawa ${ }^{6}$,

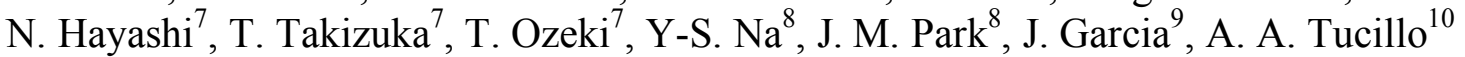 \\ ${ }^{1}$ Princeton Plasma Physics Laboratory, Princeton, NJ, USA \\ ${ }^{2}$ CEA Cadarache, Saint-Paul-Lez Durance, France \\ ${ }^{3}$ Max-Planck-Institut für Plasmaphysik, EURATOM-Assoziation, Garching, Germany \\ ${ }^{4}$ Oak Ridge National Laboratory, Oak Ridge, TN, USA \\ ${ }^{5}$ General Atomics, San Diego, CA, USA \\ ${ }^{6}$ ITER International Team, ITER Naka Joint Work Site, Naka, Ibaraki, Japan \\ ${ }^{7}$ Japan Atomic Energy Agency, Naka, Ibaraki, Japan \\ ${ }^{8}$ National Fusion Research Center, 52 Yeoeun-Dong, Yusung-Gu, Daejeon, 305-333, Korea \\ ${ }^{9}$ Universitat Politecnica de Catalunya, Barcelona, Spain \\ ${ }^{10}$ Associazione EURATOM-ENEA, CR ENEA-Frascati, Rome, Italy \\ e-mail contact of main author: ckessel@pppl.gov
}

\begin{abstract}
Integrated simulations are done to establish a physics basis, in conjunction with present tokamak experiments, for the operating modes in ITER. Simulations of the hybrid mode are done using both fixed and free-boundary 1.5D transport evolution codes including CRONOS, ONETWO, TSC/TRANSP, TOPICS, and ASTRA. The hybrid operating mode is simulated using the GLF23 energy transport model. The injected powers are limited to the negative ion neutral beam (NBI), ion cyclotron (ICRF), and electron cyclotron (EC). Several plasma parameters and source parameters are specified for the hybrid cases to provide a comparison among the simulations. Simulations of the steady state operating mode are done with the same 1.5D transport evolution codes cited above. In these cases the energy transport model is more difficult to prescribe, so that energy confinement models will range from theory based to empirically based. The injected powers include the same sources for the hybrid with the possible addition of lower hybrid (LH). These simulations will be presented and compared with particular focus on code to code results when using the same energy transport model, and within the same code when using different energy transport models
\end{abstract}

\section{Introduction}

The International Thermonuclear Experimental Reactor (ITER) project[1,2] has identified three primary operating modes for demonstrating controlled burning plasmas, motivated by experiments on existing tokamaks that demonstrate their potential for good performance. The reference operating mode is the ELMy H-mode, with $\mathrm{I}_{\mathrm{P}}=15 \mathrm{MA}, \mathrm{B}_{\mathrm{T}}=5.3 \mathrm{~T}, \mathrm{R}=6.2 \mathrm{~m}$, $\mathrm{a}=2.0 \mathrm{~m}, \kappa_{\mathrm{x}}=1.85, \delta_{\mathrm{x}}=0.5, \mathrm{P}_{\mathrm{alpha}}=80 \mathrm{MW}$, and $\mathrm{P}_{\mathrm{aux}}=40 \mathrm{MW}$, obtaining a fusion gain $(\mathrm{Q}$ $\left.=\mathrm{P}_{\text {fusion }} / \mathrm{P}_{\mathrm{aux}}\right)$ of 10 . The hybrid mode has the same geometry and toroidal field, but operates at lower plasma current, $12 \mathrm{MA}$, leading to higher safety factor and requiring lower loop voltage to drive inductive current. Present experiments on the hybrid configuration, which are heated by neutral beam injection (NBI), show that the plasma has higher energy confinement than the standard ELMy H-mode and can operate near the no wall $n=1 \beta$ limit $\left(\beta_{\mathrm{N}} \approx 3\right)$ without neo-classical tearing modes $(\mathrm{NTM})$ seriously degrading its performance. This results in higher bootstrap current further reducing the loop voltage. The high $\beta_{\mathrm{N}}$ and low loop voltage give it the potential of providing high neutron fluence (neutron wall load $x$ flattop time) for nuclear testing in ITER. The steady state (or advanced tokamak) mode has an even lower plasma current, $\sim 9 \mathrm{MA}$, and a slightly larger major radius, $6.35 \mathrm{~m}$, and a smaller minor radius, $1.85 \mathrm{~m}$, with stronger shaping $\kappa_{\mathrm{x}}=2.0, \delta_{\mathrm{x}}=0.5$. For these the noninductive current is $100 \%$ in flattop, while inductive current drive would be used in the current ramp up. The safety factor is above 1.5 throughout the plasma. Although steady state configurations can be found with $\beta_{\mathrm{N}}$ near the no wall $n=1 \beta$ limit, it is desired to demonstrate 
sustained operation above this limit with resistive wall mode feedback. Advanced tokamak experiments on present tokamaks vary widely due to the variations in the heating and current drive sources, density control, and plasma shaping.

The goals of these integrated simulations are to establish a physics basis, in conjunction with present tokamak experiments, for these operating modes in ITER. Since it is not possible to reproduce all the physics parameters of ITER plasmas simultaneously in present experiments, simulations are used to project to the ITER regime using theoretically based physics models, that are being tested against present tokamak experiments. These efforts include energy, particle, current, and momentum transport, ideal MHD, and heating and current drive in timedependent discharge simulations. In order to provide a more consistent prediction for ITER, a common set of parameters for the scenarios and a comparison (benchmarking) of the various codes has been coordinated by the Steady State Operation (SSO) topical group of the International Tokamak Physics Activity (ITPA). The simulations will be presented and compared with particular focus on code to code results when using the same energy transport model, and within the same code when using different energy transport models.

\section{Simulation Tools Used in Scenario Modeling}

The Tokamak Simulation Code (TSC) $[3,4]$ is used for the TSC/TRANSP predictive freeboundary time-dependent transport simulations, and solves the axisymmetric 2D MHDMaxwell's equations on a rectangular grid. 1D flux surface averaged transport equations are solved for energy, particles, and current density utilizing predefined transport coefficients. TRANSP $[5,6]$ is used in the "interpretive" mode, where it receives equilibrium data, ion and electron temperature profiles, density profile, and $\mathrm{Z}_{\text {eff }}$ profile from TSC, and solves flux conservation equations governing the flux surface averaged 1D transport for energy, particles, current density, and momentum. The source deposition and current drive profiles from TRANSP are then used in TSC. The source models in TRANSP include NUBEAM[5] (Monte Carlo orbit following) for neutral beam injection (NBI), TORIC4[7] (full wave) for ion cyclotron radio frequency (ICRF), TORAY [8] (ray-tracing relativistic damping) for electron cyclotron (EC), and LSC [9] (ray-tracing quasilinear 1D Fokker Planck) for lower hybrid (LH). TRANSP accounts for the fast particle populations from NBI and fusion and includes them in the ICRF damping through equivalent Maxwellians derived from their slowing down distributions.

CRONOS is a suite of numerical codes for the predictive/interpretative simulation of a full tokamak discharge [10]. It integrates, in a modular structure, a 1-D transport solver with general 2-D magnetic equilibria, self-consistently computed by the HELENA code [11], including several heat, particle and impurities transport models, as well as heat, particle, current and momentum sources. The source modules used in the simulations included here are the following: SINBAD [12] for NBI heating and current drive, PION [13] for ICRH, REMA [14] for EC ray tracing, with a linear estimate of the ECCD efficiency [15], Delphine [16] for LH ray tracing (including 2D Fokker-Planck evaluation of LHCD efficiency), and SPOT (an orbit following Monte Carlo code) for the alpha particles distribution function [17].

TOPICS (TOkamak Prediction and Interpretation Code System) $[18,19,20]$ is an integrated code system for the predictive simulation and the interpretation of the experimental data. The base part of TOPICS is a 1.5D transport code for the electron and ion temperatures, the densities of any species, and the poloidal magnetic field, self-consistently with the free- 
boundary equilibrium of arbitrary shape and the external sources of the heat, particles, momentum and current. The NB and EC heating profiles are prescribed here for simplicity. The ICRF heating profile is taken from the TORIC4 results provided by the TSC/TRANSP simulations. The driven current profiles of NBCD and ECCD are calculated with the Mikkelsen and Singer's approximation [21] and with an empirical formula of the current drive efficiency, $\eta_{\mathrm{ECCD}}=0.055\left(\mathrm{~T}_{\mathrm{e}}[\mathrm{keV}]\right)^{0.5}\left[10^{20} \mathrm{~A} / \mathrm{Wm}^{2}\right][22]$, respectively.

The ASTRA code [23] solves coupled, time-dependent, 1-D transport equations for particles, heat, and current, as well as 2-D MHD fixed boundary equilibrium (ESC employed to calculate MHD equilibrium) self-consistently with a realistic tokamak geometry.

Here up-down symmetry is assumed. The NBI package [24] is embedded in ASTRA for the calculation of NBI heating and current drive. A model developed by Kim [25] is employed to calculate the bootstrap current in the plasma. For the work done here the other source deposition profiles are prescribed.

The ONETWO transport code [26,27] solves the flux surface averaged transport equations for energy, particles, toroidal rotation, current density and equilibrium evolution with selfconsistent source and sink calculation. Predictive simulation from ONETWO with the GLF23 transport model employs a globally convergent nonlinear solution method based on, modified Newton, trust region, and steepest descent methods with an improved computational efficiency by a recent parallelization of ONETWO. The stationary state of ITER hybrid scenario with fully penetrated current profile is obtained by interleaving time stepping calculation of all the transport equations and one step solution of current evolution. The source models used in ONETWO are the ray tracing codes TORAY-GA for EC and CURRAY[28] for ICRF, and the Monte Carlo code NUBEAM[5] for neutral beam heating and current drive.

\section{ITER Hybrid Scenarios}

Simulations of the hybrid are done using both fixed and free-boundary 1.5D transport evolution codes including CRONOS, ONETWO, TSC/TRANSP, TOPICS, and ASTRA. The hybrid operating mode is simulated using the GLF23[29] core energy transport model. The DT fuel ion ratio is assumed to be 50-50. The impurities are Be and Ar, with assumed fractions of $2 \%$ and $0.12 \%$, respectively. The impurity density profiles are forced to be the same as the electron density, while the impurity temperatures are set equal to the main ion temperatures The fuel ion density profiles are determined from quasi-neutrality. The electron density profile is prescribed to give a very broad profile with a peak to volume average of about 1.05, and the peak value and separatrix value are specified. For these simulations the pedestal parameters are specified as $\rho_{\text {ped }}=0.925, \mathrm{n}_{\text {ped }}=\mathrm{n}(\rho=0.925)=\mathrm{n}(0)=$ $0.85 \times 10^{20} / \mathrm{m}^{3}, \mathrm{~T}_{\text {ped }}$ is set to $5 \mathrm{keV}$. The plasma density profile assumed is shown in Fig. 1, and has its value linearly reduced from the maximum value at $\rho_{\text {ped }}=0.925$ to $0.35 \mathrm{x} \mathrm{n}(0)$ at the seperatrix, while all temperatures are forced to be $200 \mathrm{eV}$ at the separatrix. The ratio of the effective particle to energy confinement time $\left(\tau_{\mathrm{p}}{ }^{*} / \tau_{\mathrm{E}}\right)$ is enforced to be 5.0. The injected powers are limited to the negative ion NBI ( $1 \mathrm{MeV}, 33 \mathrm{MW}$, steered to full off-axis), ICRF heating $\left(20 \mathrm{MW}, 53 \mathrm{MHz}, 2^{\text {nd }} \mathrm{T}\right.$ harmonic) and EC heating and current drive $(170 \mathrm{GHz}, 20$ $\mathrm{MW}$, midplane launch steered for off-axis CD).

Shown in Figs. 1 and 2 are the temperature, density, safety factor, external power deposition and toroidal current density profiles from the various ITER hybrid simulations using NBI and 
ICRF heating. All simulations use the GLF23 core transport model where the GLF23 diffusivities are added to the neoclassical values, and the poloidal rotation is assumed to be neoclassical [30]. The TOPICS simulation uses the CDBM05 core transport model[31]. The TSC/TRANSP and CRONOS simulations only solve for the energy channels $\left(T_{e}\right.$ and $\left.T_{i}\right)$, while prescribing the plasma toroidal rotation calculated by assuming that $\chi_{\text {mom }}=\chi_{i}$ in TRANSP, and neglecting the $\alpha$-stabilization. The ONETWO simulations solve the energy $\left(T_{e}\right.$ and $\left.T_{i}\right)$ equations and the toroidal momentum equation $\left(\Omega_{R}\right)$ using the GLF23 energy and momentum transport model. The toroidal momentum diffusivity is added to an ad hoc enhancement, taken in this case to be twice the neoclassical ion thermal diffusivity[27]. In the ASTRA calculations the toroidal velocity is assumed as $\mathrm{v}_{\text {tor }}=2.5 \times 10^{4} \times \mathrm{T}_{\mathrm{i}}(\mathrm{keV})$ assuming $\chi_{\text {mom }}$ is proportional to $\chi_{i}[32]$. ICRF deposition profiles are taken from TORIC4 results provided by the TSC/TRANSP simulations. EC deposition profiles are taken from TORAY results provided by the ONETWO simulations. In the TOPICS hybrid simulation the $\mathrm{E} \times \mathrm{B}$ shear stabilization on the anomalous transport is not taken into account. In order to produce an $\mathrm{H}$-mode pedestal, the transport is reduced to the neoclassical level in the peripheral region $(\rho>0.925$ according to the guideline). In the TOPICs cases a sawtooth oscillation is simulated with Kadomtsev's model with a time interval of $\tau_{\mathrm{sw}}=10 \mathrm{~s}$ [33].

Overall, the agreement on electron and ion temperature profiles is reasonable, however, the differences inside of $\rho=0.25$ are as large as $10 \mathrm{keV}$. The various codes have used the GLF23 model with slightly different settings ( $\alpha$ stabilization on or off, toroidal momentum transport on or off) indicating that very strict prescriptions for the use of GLF23 will be required to understand the implimentation differences of the codes. The CDBM05 model appears to give similar results. The safety factor profiles are similar, with the differences well correlated to the differences in the toroidal current density profiles. In order to reproduce the behavior of experimental hybrid discharges in which q remains above 1.0 and does not sawtooth, the $1.5 \mathrm{D}$ transport codes will require an artificial model that controls the current profile. The significant weakening of the magnetic shear near the plasma edge due to large edge bootstrap current is a concern for ideal MHD peeling modes, and the differences in the codes bootstrap current there is as high as a factor of 2. The TOPICS simulation has imposed a sawtooth model which flattens the $\mathrm{q}$ and current density profiles. The total external heating profiles for electrons and ions, which include both the NBI and ICRF heating, are in fairly good agreement, with most differences attributable to well known modeling differences (linear ray-tracing versus full wave for ICRF, or Monte Carlo orbit following versus Fokker-Planck for NBI), although some differences are linked to slightly different ICRF frequencies used (52.5 versus $56 \mathrm{MHz}$, for example) and inclusion of impurity and fast particle effects.

Shown in Figs. 3 and 4 are hybrid simulations done adding 20 MW of EC heating and offaxis current drive, using the midplane launchers, to the previous hybrid scenario. The same approaches and prescriptions are used for these simulations as given above. It is clear that $T_{e}$ is higher under the direct electron heating, and $T_{i}$ also shows a significant increase inside $\rho=$ 0.25 . The difference among the temperature profiles is smaller, although the behavior near the magnetic axis is similar to the NB+IC hybrid cases. One reason for the close agreement for the ion temperature profiles may be that the additional power has weakened the stabilization mechanisms sufficiently to basically give the unstabilized turbulent result, which the various codes should give very similar results for. The q profiles show some distortion 
associated with the ECCD inside $\rho=0.4$, however the net driven current is insufficient to stop $q(0)$ from dropping well below 1.0. The toroidal current densities show the ECCD features. The electron external power deposition profiles show that more work is required to get consistent results among the simulations for EC.

\section{ITER Steady State Scenarios}

Simulations of the steady state operating mode are done with the same 1.5D transport evolution codes cited above. In these cases the energy transport model is more difficult to prescribe since the models, used for H-mode plasmas (like the ELMy H-mode and hybrid) that are dominated by ExB rotational shear stabilization, have deficiencies when applied to reversed shear $(\mathrm{dq} / \mathrm{dr}<0)$, and high pressures (Shafranov shift). Therefore the energy confinement prescriptions will range from theory based to empirically based models. The available heating and CD sources are the same as for the hybrid, but with the addition in some cases of 20-35 MW of lower hybrid power for far off-axis CD. Shown in Figs. 5 and 6 are the electron and ion temperature, density and power deposition profiles, and toroidal current density profiles with their non-inductive contributions.

The TSC/TRANSP simulation utilized the NBI (16.5 MW steered partially off-axis), ICRF (20 MW heating only) and $\mathrm{LH}\left(35 \mathrm{MW}, \mathrm{n}_{\|}=2.15, \Delta \mathrm{n}_{\|}=0.2,5.0 \mathrm{GHz}\right)$ assuming a factor of 2 CD enhancement found from 2D Fokker Planck LH simulations [34]. The temperature pedestal was set at $2 \mathrm{keV}$, and analytic thermal diffusivities were used to produce ITB-like temperature profiles, while the density profile was prescribed. The impurities were $2 \% \mathrm{Be}$ and $0.1 \%$ Ar, giving $Z_{\text {eff }}$ of 1.65 . The total plasma current is $8.0 \mathrm{MA}$, of which $4.8 \mathrm{MA}$ is bootstrap, 2.1 MA is from $\mathrm{LH}$, and $0.87 \mathrm{MA}$ is from NBI. The $\beta_{\mathrm{N}}$ is 3.1 , the $\mathrm{P}_{\alpha}$ is $78 \mathrm{MW}$, and li(1) is 0.6 . The peak density is $0.78 \times 10^{20} / \mathrm{m}^{3}, \mathrm{n} / \mathrm{n}_{\mathrm{Gr}}$ is 1.05 , and $\mathrm{H}_{98}$ is 1.73 . Another case was done with a slightly more peaked density, $\mathrm{n}(0) /<\mathrm{n}>=1.2$, which yielded similar parameters but required $25 \mathrm{MW}$ of $\mathrm{LH}$ power.

For the TOPICS steady-state simulation, the following conditions and models are employed. For the anomalous transport, we apply a modified CDBM model to weaken the $\alpha$ stabilization in the function F, which has been validated in JT-60U strongly-RS plasmas with a box-type ITB [19]. The ExB shear stabilization on the anomalous transport is not taken into account. The NB and EC heating profiles are prescribed for simplicity. The driven current profiles of NBCD and ECCD are calculated with a current drive efficiency of 0.4 $\left[10^{20} \mathrm{~A} / \mathrm{Wm}^{2}\right]$ for a well optimized system [35] and with an empirical formula of the current drive efficiency, $\eta_{\mathrm{ECCD}}=0.055\left(\mathrm{~T}_{\mathrm{e}}[\mathrm{keV}]\right)^{0.5}\left[10^{20} \mathrm{~A} / \mathrm{Wm}^{2}\right][22]$, respectively. The plasma current is $9 \mathrm{MA}$, composed of 4.6 MA of bootstrap, 2.48 MA of NBCD, and 1.76 MA of ECCD. The $\mathrm{P}_{\alpha}$ reaches $63 \mathrm{MW}, \beta_{\mathrm{N}}$ is $3.5, \mathrm{li}(1)$ is $0.58, \mathrm{H}_{98}$ is 1.86 , and $\mathrm{n}(0)=0.76 \times 10^{20}$ $/ \mathrm{m}^{3}$.

The CRONOS simulations of an ITER steady-state regime have taken full advantage of the built-in feedback algorithms in CRONOS. A "search optimization control" (SOC) algorithm has been used [36] which tries to optimize the Q factor imposing, at the same time, constraints such as loop voltage lower than a given value, Greenwald fraction less than a given value, $\beta_{\mathrm{N}}$ less than $4 \times \mathrm{l}_{\mathrm{i}}$. Using such an algorithm is a practical means of finding an appropriate advanced regime of operation for ITER. The simulation shown here is obtained using $20 \mathrm{MW}$ of LH power at $5 \mathrm{GHz}, \mathrm{n}_{\|}=2, \mathrm{ICRH}$ power $\approx 16.5 \mathrm{MW}, \mathrm{NBI}$ power $\approx 6 \mathrm{MW}$. The transport model [37] imposes an energy confinement time in agreement with the pure 
gyroBohm DS03 global scaling [38], with an additional shear function to allow the formation of an ITB for negative shear. The total plasma current is $8.5 \mathrm{MA}$, with approximately $50 \%$ of bootstrap fraction and $75 \%$ of total non-inductive current, at $\mathrm{f}_{\mathrm{G}} \sim 0.8$ and $\mathrm{Q} \sim 7$. An ITB is present at normalized radius $\sim 0.6$, with $\mathrm{H} \sim 1.4$.

The ONETWO simulations of an ITER steady state scenario utilizes $33 \mathrm{MW}$ of NBI, $20 \mathrm{MW}$ of ICRF (co-CD phasing), and $20 \mathrm{MW}$ EC (top launch steered for off-axis). The GLF23 core transport model is used. The plasma current is $9 \mathrm{MA}$, with 6.2 MA from bootstrap, 2.11 MA from NBCD, and 0.74 MA from FWCD and ECCD. The $\beta_{\mathrm{N}}$ reaches 2.5 , the $\mathrm{P}_{\alpha}$ is $83 \mathrm{MW}$, the safety factor is monotonic with $\mathrm{q}(0) \approx 1.45$. The peak density is $0.85 \times 10^{20} / \mathrm{m}^{3}$. The pedestal temperature for this case is about $8 \mathrm{keV}$, however when the pedestal temperature is lowered to $7 \mathrm{keV}$ a strong transport barrier forms making $\mathrm{T}_{\mathrm{i}}(0)$ increase from 20 to $40 \mathrm{keV}$, $\mathrm{T}_{\mathrm{i}} / \mathrm{T}_{\mathrm{e}}$ is about 1.5, the $\mathrm{P}_{\alpha}$ rises to $112 \mathrm{MW}$, and $\beta_{\mathrm{N}}$ reaches 3.8. This results in a negative central shear q profile out to $\rho \approx 0.6$. These results are being optimized and are not shown.

\section{Conclusions and Future Work}

The SSO topical group of the ITPA has begun to examine integrated simulation benchmarks for the hybrid scenario in ITER. These activities involve the comparison of 1.5D core transport modeling assumptions, source physics modeling assumptions, as well as numerous peripheral physics modeling. Initial results indicate that very strict guidelines will need to be imposed on the application of GLF23, for example, to make useful comparisons. Issues include, transport channels included in the simulations, use of $\alpha$-stabilization term, rotation stabilization features, and approaches used to stabilize the algorithm. The source models vary widely among the simulations, and continued upgrading of the codes is necessary. Since this involves a tradeoff between computational speed and physics fidelity, we must make some assessments of how good is good enough for a given source application. Finally there are a number of peripheral physics models that should be examined, some of which include fusion power production, bootstrap current, treatment of fast particles, and treatment of impurities. The SSO modeling group is also pursuing new capabilities, with other ITPA groups, to improve the consistency of the simulations including pedestal models, core to divertor models, more routine ideal and fast particle MHD analysis, and comparison with gyrokinetic simulations. In the future, benchmark simulations will also begin for the ITER steady state scenarios.

The first author of this work is supported by DoE Contract No. DE-AC02-76-CH0-3073.
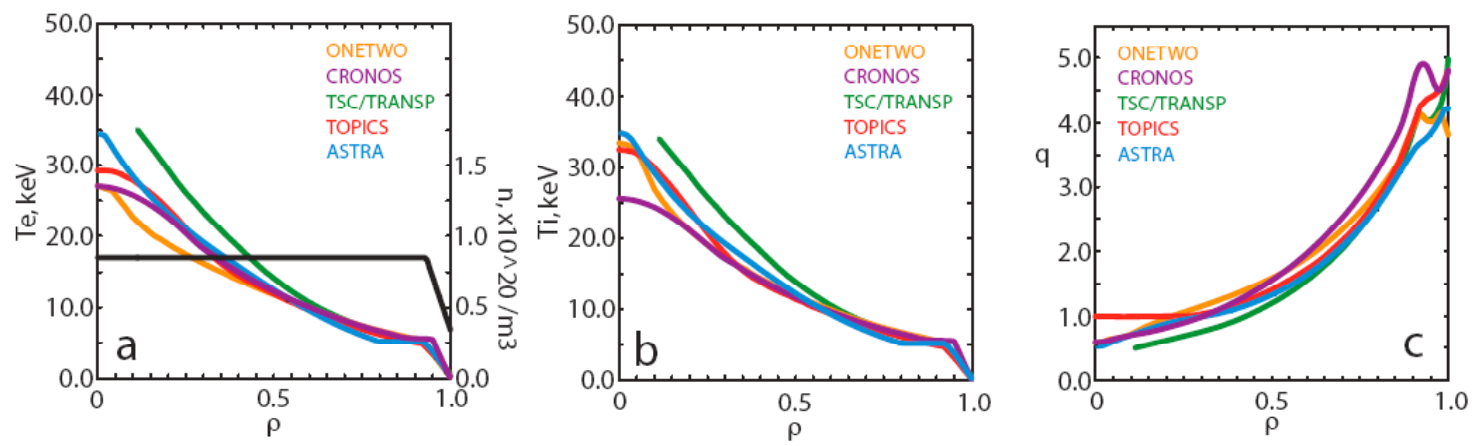

Figure 1. Electron temperature profiles and density profile (a), ion temperature profiles (b), safety factor profiles (c), for the NB+IC ITER Hybrid simulations. 

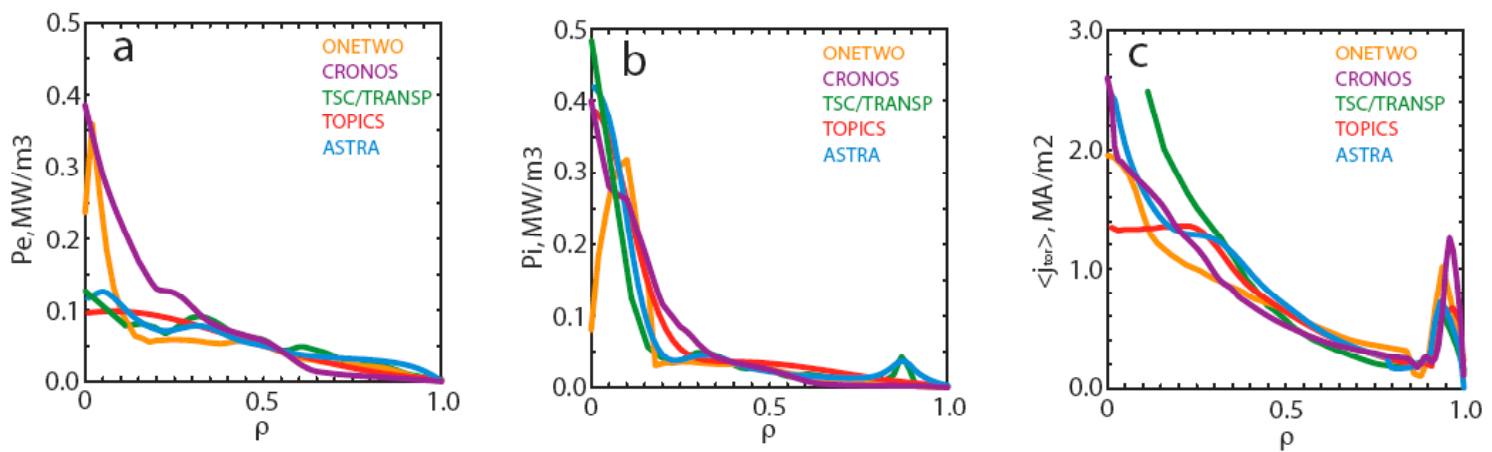

Figure 2. External power deposition profiles to electrons (a) and ions (b) and the toroidal current density (c) for the NB+IC ITER Hybrid simulations.
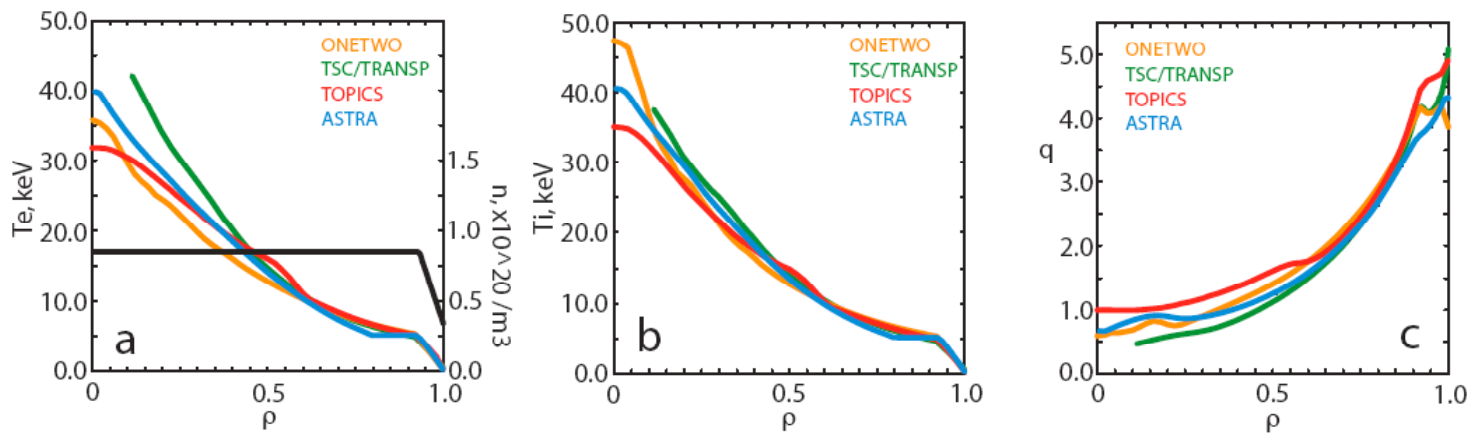

Figure 3. Electron temperature profiles and density profile (a), ion temperature profiles (b), safety factor profiles (c), for the $N B+I C+E C$ ITER Hybrid simulations.
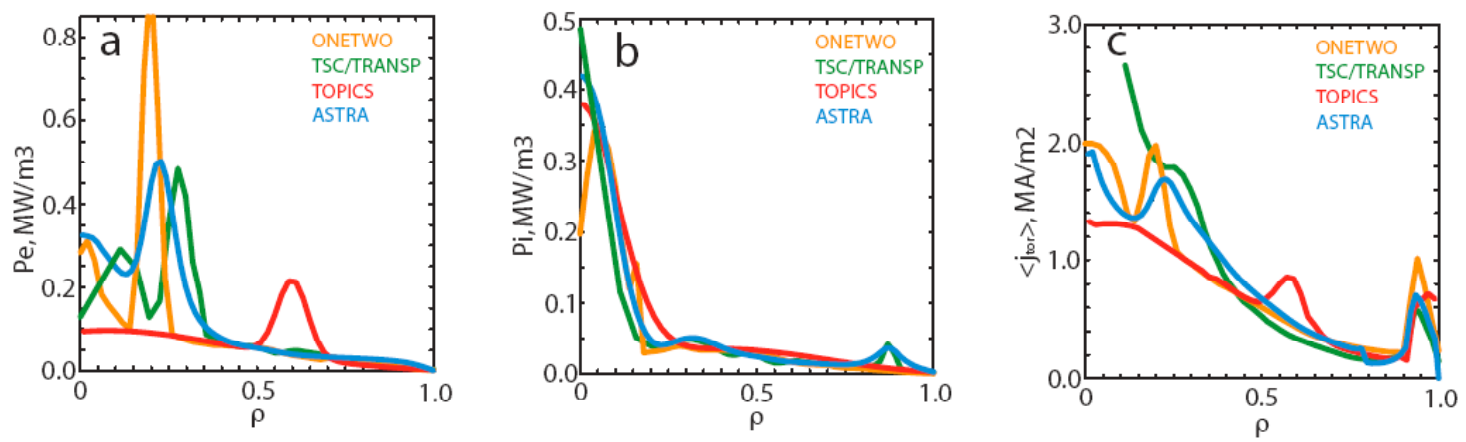

Figure 4. External power deposition profiles to electrons (a) and ions (b) and the toroidal current density (c) for the NB+IC ITER Hybrid simulations.
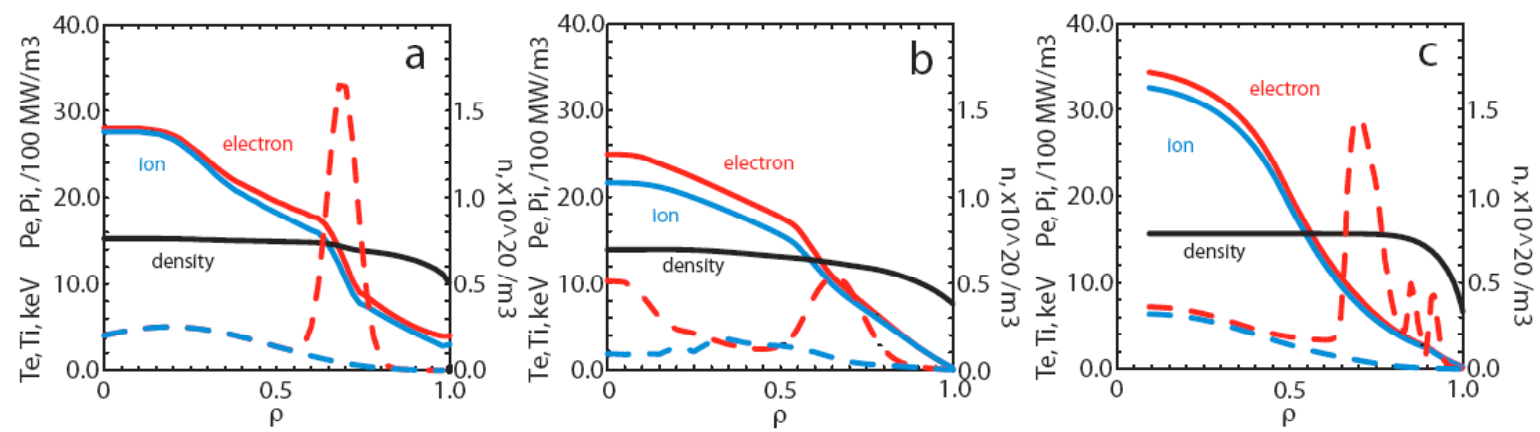

Figure 5. Electron and ion temperature, density, and external power deposition profiles for Steady State ITER simulations, (a) TOPICS (NB+EC), (b) CRONOS $(N B+I C+L H)$, and (c) TSC/TRANSP $(N B+I C+L H)$. 

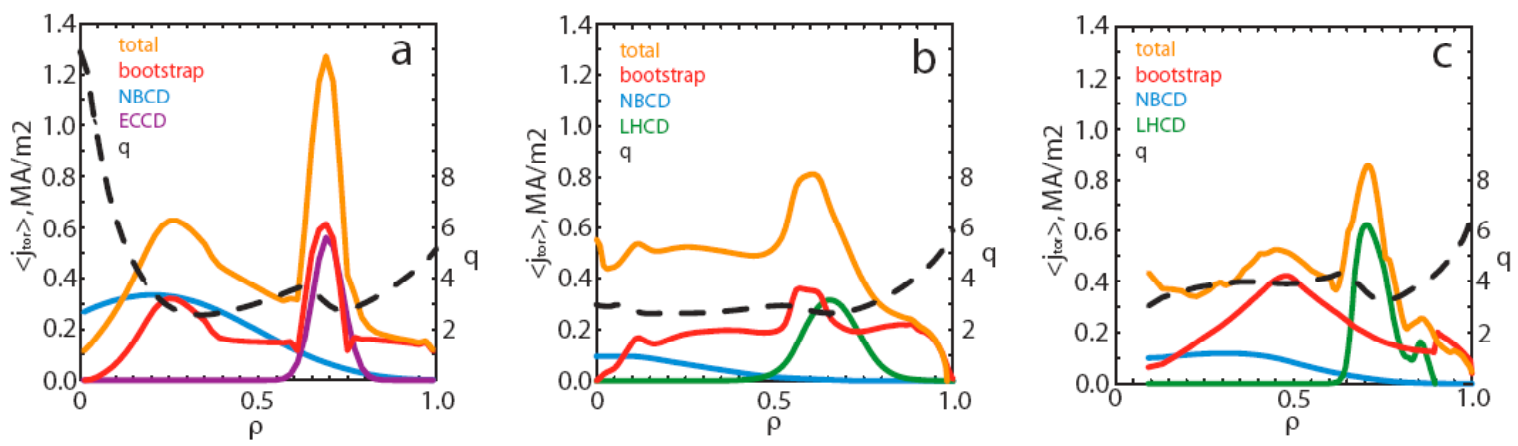

Figure 6. Safety factor and toroidal current density profiles and its contributions for Steady State ITER simulations, (a) TOPICS $(N B+E C)$, (b) CRONOS $(N B+I C+L H)$, and (c) TSC/TRANSP $(N B+I C+L H)$.

\section{References}

[1] TECHNICAL BASIS FOR THE ITER-FEAT OUTLINE DESIGN, ITER-EDA Documentation Series No.

19, IAEA, Vienna, 2000. (also http://www.iter.org).

[2] ITER PHYSICS BASIS, Nucl. Fusion, 39, 1999.

[3] S. C. JARDIN et al 1983 J. Comput. Phys. 66481.

[4] C. E. KESSEL et al 2006 Phys. Plas. 13056108.

[5] R. J. GOLDSTON et al 1981 J. Comput. Phys. 4361.

[6] R. V. BUDNY et al 1992 Nucl. Fusion 32429.

[7] M. BRAMBILLA 1996 A Full Wave Code for Ion Cyclotron Waves in Toroidal Plasmas, Rep. IPP 5/66, Max-Planck-Institut fur Plasmaphysik, Garching.

[8] A. H. KRITZ et al 1982 Heating in Toroidal Plasmas, Proc. $3^{\text {rd }}$ Joint Varenna-Grenoble Int. Symp. Grenoble, 1982, Vol. 2 CEC, Brussels (1982) 707.

[9] D. W. IGNAT et al 1994 Nucl. Fusion 34837.

[10] V. BASIUK et al. 2003 Nucl. Fusion 43822.

[11] G. HUYSMANS et al. CP90 Conf. Comp. Physics (World Scientific 1991) 371.

[12] Y. FENG et al. 1995 Comp. Phys. Comm. 88161.

[13] L.G. ERIKSSON et al. 1993 Nucl. Fus. 331037.

[14] V. KRIVENSKI et al 1985 Nucl. Fus. 25127.

[15] Y.R. LIN-LIU et al. 2003 Phys. Plasmas 104065.

[16] F. IMBEAUX, Y. PEYSSON 2005 Plasma Phys. Contr. Fus. 472041.

[17] M. SCHNEIDER et al 2005 Plasma Phys. Contr. Fus. 472087.

[18] SHIRAI H. et al. 2000 Plasma Phys. Control. Fusion 421193.

[19] HAYASHI N., TAKIZUKA T. AND OZEKI T. 2005 Nucl. Fusion 45933.

[20] HAYASHI N. et al., this conference TH/4-2.

[21] MIKKELSEN D.R. AND SINGER C.E. 1983 Nucl. Technol./Fusion 4237.

[22] HARVEY R.W. et al. 1997 Nucl. Fusion 3769.

[23] PEREVERZEV G. et al 2002 IPP-Report IPP 5/98.

[24] POLEVOI A et al 1997 JAERI-Data/Code 97-014.

[25] KIM Y B et al 1991 Phys. Fluids B 32050.

[26] H. E. ST. JOHN et alPlasma Physics and Controlled Nuclear Fusion Research 1994, in Proceedings of the

15th IAEA Conference, Seville, 1994 IAEA, Vienna, 1994), Vol. 3, p. 603.

[27] M. MURAKAMI et al 2005 Nuclear Fusion 451419.

[28] T. K. MAU et al 1992, EPS Topical Conf. Abstracts on Radiofrequency Heating and Current Drive in Fusion Devices (Brussels, 1992) p 181.

[29] J. E. KINSEY et al 200512 052503-1.

[30] STAEBLER G M et al 1997 Nucl. Fusion 37287.

[31] HONDA M. AND FUKUYAMA A. 2006 Nucl. Fusion 46580.

[32] KALLENBACH A et al 1991 Plasma Phys. Control. Fusion 33595.

[33] KADOMTSEV B.B. 1975 Sov. J. Plasma Phys. 1389.

[34] P. BONOLI et al, this conference IT/P1-2.

[35] DE ESCH H.P.L. et al. 1995 Fusion Eng. Des. 26589.

[36] V. BASIUK et al., $33^{\text {rd }}$ EPS Conference, Rome June 19-23, 2006, P1.125.

[37] J.F. ARTAUD et al., 32nd EPS Conference, Tarragona, June 27 - July 1, 2005, P1.035.

[38] C. PETTY et al., 2003 Fus. Sci. Techn. 431. 